\title{
Kauffman bracket skein module of a connected sum of 3-manifolds
}

\author{
Józef H. Przytycki
}

\begin{abstract}
.
We show that for the Kauffman bracket skein module over the field of rational functions in variable $A$, the module of a connected sum of 3-manifolds is the tensor product of modules of the individual manifolds.
\end{abstract}

\section{The main theorem.}

We recall in this section the definition of the Kauffman bracket skein module (KBSM) and formulate the main result of the paper.

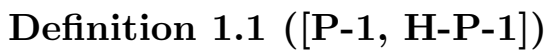

Let $M$ be an oriented 3-manifold, $R$ a commutative ring with identity and $A$ its invertible element. Let $\mathcal{L}_{f r}$ be the set of unoriented framed links in $M$, including the empty link $\emptyset$. Let $S$ be the submodule of $R \mathcal{L}_{f r}$ generated by skein expressions $L_{+}-A L_{0}-A^{-1} L_{\infty}$, where the triple $L_{+}, L_{0}, L_{\infty}$ is shown in Fig.1.1, and $L \sqcup T_{1}+\left(A^{2}+A^{-2}\right) L$, where $T_{1}$ denotes the trivial framed knot. We define the Kauffman bracket skein module (KBSM), denoted by $\mathcal{S}(M ; R, A)$, as the quotient $\mathcal{S}(M ; R, A)=R \mathcal{L}_{f r} / S$.

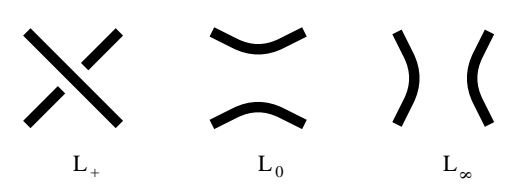

Fig. 1.1.

\footnotetext{
${ }^{1}$ The standard notation for the $\operatorname{KBSM}$ is $\mathcal{S}_{2, \infty}(M ; R, A)$, $\mathrm{P}-1$, $\mathrm{H}-\mathrm{P}-1$, but in this paper we do not discuss skein modules other than $\operatorname{KBSM}$ so for simplicity we drop $(2, \infty)$ from the notation.
} 
Notice that $L^{(1)}=-A^{3} L$ in $\mathcal{S}(M ; R, A)$, where $L^{(1)}$ denotes a link obtained from $L$ by one positive twist of the framing of $L$. We call this the framing relation. For the sake of shortness of notation, we will often $\operatorname{drop}(R, A)$ from $\mathcal{S}(M ; R, A)$ and write simply $\mathcal{S}(M)$, as long as it unambiguous.

Theorem 1.2 Assume that $\left(A^{k}-1\right)$ is invertible in $R$ for any $k>0$. Then

$$
\mathcal{S}\left(M_{1} \# M_{2}\right)=\mathcal{S}\left(M_{1}\right) \otimes \mathcal{S}\left(M_{2}\right)
$$

where $M_{1} \# M_{2}$ denotes the connected sum of compact 3-manifolds $M_{1}$ and $M_{2}$.

In particular we have:

Corollary 1.3 If $R$ is a field of rational functions in variable $A, \mathcal{F}(A)$, or $R$ is a field of complex numbers, $C$, and $A$ is not a root of unity, then $\mathcal{S}\left(M_{1} \# M_{2}\right)=\mathcal{S}\left(M_{1}\right) \otimes \mathcal{S}\left(M_{2}\right)$.

\section{Basic properties of skein modules}

Below we list some elementary properties of KBSM (which also hold for other skein modules), [P-1] (compare also [H-P-1, $\mathrm{P}-3]$ ).

Proposition 2.1 (1) An orientation preserving embedding of 3-manifolds $i: M \rightarrow N$ yields the homomorphism of skein modules $i_{*}: \mathcal{S}(M) \rightarrow \mathcal{S}(N)$. The above correspondence leads to a functor from the category of 3-manifolds and orientation preserving embeddings (up to ambient isotopy) to the category of $R$-modules (with a specified invertible element $A \in R$ ).

(2) (i) If $N$ is obtained from $M$ by adding a 3-handle to it (i.e. capping off a hole so that $\left.M=N \# D^{3}\right)$, and $i: M \rightarrow N$ is the associated embedding, then $i_{*}: \mathcal{S}(M) \rightarrow$ $\mathcal{S}(N)$ is an isomorphism.

(ii) If $N$ is obtained from $M$ by adding a 2-handle to it, and $i: M \rightarrow N$ is the associated embedding, then $i_{*}: \mathcal{S}(M) \rightarrow \mathcal{S}(N)$ is an epimorphism.

(3) If $M_{1} \sqcup M_{2}$ is the disjoint sum of 3-manifolds $M_{1}$ and $M_{2}$ then

$$
\mathcal{S}\left(M_{1} \sqcup M_{2}\right)=\mathcal{S}\left(M_{1}\right) \otimes \mathcal{S}\left(M_{2}\right)
$$

(4) (Universal Coefficient Property)

Let $r: R \rightarrow R^{\prime}$ be a homomorphism of rings (commutative with 1). We can think of $R^{\prime}$ as an $R$ module. Then the identity map on $\mathcal{L}_{f r}$ induces the isomorphism of $R^{\prime}$ (and $R$ ) modules:

$$
\bar{r}: \mathcal{S}(M ; R, A) \otimes_{R} R^{\prime} \rightarrow \mathcal{S}\left(M ; R^{\prime}, r(A)\right)
$$


(5) If $F$ is a surface, then the $K B S M \mathcal{S}(F \times I)$ is a free $R$-module with basis $B(F)$ consisting of links on $F$, up to ambient isotopy of $F$, without contractible components (but including the empty link).

Results in Proposition 2.1 are well known; compare $\mathrm{P}-1$, H-P-1, $\mathrm{P}-\mathrm{S}, \mathrm{P}-3$. We clarify some points of them below:

(1) If $i: M \rightarrow N$ is an orientation reversing embedding then $i_{*}$ is a $Z$-homomorphism and $i_{*}(A w)=A^{-1} i_{*}(w)$.

(2) (i) It holds because the co-core of a 3-handle is 0-dimensional.

(ii) It holds because the co-core of a 2-handle is 1-dimensional.

(3) This is a consequence of the well known property of short exact sequences, [B]]:

If $0 \rightarrow A^{\prime} \rightarrow A \rightarrow A^{\prime \prime} \rightarrow 0$ and $0 \rightarrow B^{\prime} \rightarrow B \rightarrow B^{\prime \prime} \rightarrow 0$ are short exact sequences of $R$-modules then $0 \rightarrow A^{\prime} \otimes B+A \otimes B^{\prime} \rightarrow A \otimes B \rightarrow A^{\prime \prime} \otimes B^{\prime \prime} \rightarrow 0$ is a short exact sequence.

(4) This important fact follows easily from right exactness of the tensor functor (applied to a short exact sequence) and from the "five lemma" (see for example C-E]).

(5) This applies in particular to a handlebody, because $H_{n}=P_{n} \times I$, where $H_{n}$ is a handlebody of genus $n$ and $P_{n}$ is a disc with $n$ holes.

\section{Outline of the proof of the main theorem.}

1. Any compact 3-dimensional manifold can be obtained from a handlebody by adding 2 and 3-handles to it. The KBSM of a handlebody is a well understood free module (Prop. 2.1(5)), adding a 3-handle does not change the module (Prop. 2.1(2)(i)) and adding a 2-handle gives new relations to the skein module, but no new generators (Prop. 2.1(2)(ii)).

2. If a 2-handle is added, all new relations are obtained by sliding links along the 2-handle (Lemma 4.1). There is usually an infinite collection of relations, but in the case of a 2-handle added along a meridian curve (creating $S^{2}$ ), we prove that the relations form a "controllable" sequence, which, over the field $\mathcal{F}(A)$, allows us to reduce all curves cutting the sphere, but not more.

3. The embedding $j: M_{1} \# D^{3} \sqcup M_{2} \# D^{3} \rightarrow M_{1} \# M_{2}$ yields an epimorphism of the KBSM. To see that every link in $\mathcal{S}\left(M_{1} \# M_{2}\right)$ is in the image, it suffices to consider relations given by very simple slidings (Fig. 6.1), using the "second side" of $S^{2}$. We 
use also the fact that $A^{k}-1$ is invertible in $R$. $\sqcup$ denotes the disjoint sum. The connected sum $M \# D^{3}$ is a manifold obtained from $M$ by cutting off a hole in $M$. In particular, by Proposition 2.1(2)(i), the skein modules of $M$ and $M \# D^{3}$ coincide.

4. We will start the proof of Theorem 1.2, by considering the case of $M_{1}$ and $M_{2}$ being handlebodies; $M_{1}=H_{n}, M_{2}=H_{m}$. $H_{n} \# H_{m}$ is equal to $H_{n+m}$ with a 2-handle added along the boundary of the meridian disc separating $H_{n}$ from $H_{m}$. We show that the embedding $i: H_{n} \sqcup H_{m} \rightarrow H_{n} \# H_{m}$ yields an isomorphism of skein modules assuming that $A^{k}-1$ is invertible in $R$. For this we show that all sliding relations are generated by slidings of Fig. 5.1.

5. We generalize 4 . by considering any $M_{1} \# M_{2}$ and observing that $M_{1} \# M_{2}$ is obtained from $H_{n} \# H_{m}$ by adding 2-handles to $H_{n}$ or $H_{m}$.

6. In steps 4 and 5 we have to show that even a very complicated sliding (say, a curve in $H_{n}$ first being pushed to $H_{m}$ and back several times and only then slid), can be reduced to slidings of Fig. 5.1 or slidings taking place totally in $H_{n}$ or $H_{m}$ (compare Lemma 6.1).

\section{Handle sliding lemma.}

\section{Lemma 4.1}

Let $(M, \partial M)$ be a 3-manifold with the boundary $\partial M$, and let $\gamma$ be a simple closed curve on the boundary. Let $N=M_{\gamma}$ be the 3-manifold obtained from $M$ by adding a 2-handle along $\gamma$. Furthermore let $\mathcal{L}_{\text {fr }}^{\text {gen }}$ be a set of framed links in $M$ generating $\mathcal{S}(M)$.

Then $\mathcal{S}(N)=\mathcal{S}(M) / J$, where $J$ is the submodule of $\mathcal{S}(M)$ generated by expressions $L-$ $\operatorname{sl}_{\gamma}(L)$, where $L \in \mathcal{L}_{\text {fr }}^{\text {gen }}$ and $\operatorname{sl}_{\gamma}(L)$ is obtained from $L$ by sliding it along $\gamma$ (i.e. handle sliding).

Proof: Let $S^{1} \times[-1,1]$ be a tubular neighborhood of $\gamma$ in $\partial M\left(\gamma=S^{1} \times\{0\}\right)$, and consider a 2-handle added along $\gamma$, that is $D^{2} \times D^{1}$ and a homeomorphism $\phi: \partial D^{2} \times D^{1} \rightarrow S^{1} \times[-1,1]$. Then $N=M \bigcup_{\phi} D^{2} \times D^{1}$. Let $f: M \rightarrow N$ be a natural embedding, then $f_{*}: \mathcal{S}(M) \rightarrow \mathcal{S}(N)$ is an epimorphism, Prop. 2.1(2)(ii) (any link in $N$ can be pushed (ambient isotoped) to $M$ ). Furthermore any skein relation can be performed in $M$. The only difference between KBSM of $M$ and $N$ lies in the fact that some nonequivalent links in $M$ can be equivalent in $N$; the difference lies exactly in the possibility of sliding a link in $M$ along the added 2-handle (that is $L$ is moving from one side of the co-core of the 2-handle to another); compare Fig.4.1. The proof of Lemma 4.1 is completed. 

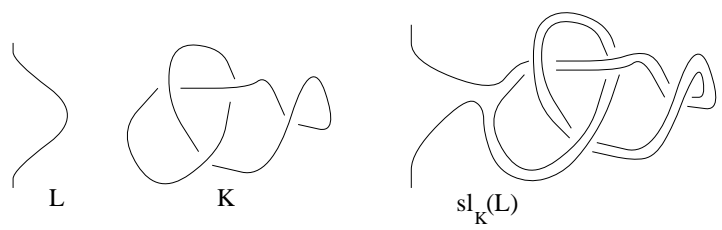

Fig. 4.1.

Lemma 4.1 allows us to write an (infinite) presentation of the Kauffman bracket skein module of any compact 3-manifold, using Heegaard decomposition and knowledge of the module for handlebodies; Proposition 2.1(5). This general presentation is not satisfactory and in some cases we can write a simpler presentation.

\section{Epimorphism}

We show in this section that for a connected sum $M_{1} \# M_{2}$, and $\left(A^{k}-1\right)$ invertible in $R$, $\mathcal{S}\left(M_{1} \# M_{2}\right)$ is generated by links, components of which are in $M_{1}$ or $M_{2}$.

The above fact follows from the slightly more general proposition which we prove below.

Proposition 5.1 Let $M$ be an oriented 3-manifold, $D$ a meridian disk in $M$, that is a properly embedded 2-disk in $M$, and $\gamma=\partial D$. If $R$ is a ring with $\left(A^{k}-1\right)$ invertible for any $k$ then the embedding $j:(M-D) \rightarrow M_{\gamma}$, where $M_{\gamma}$ is obtained from $M$ by adding a 2-handle along $\gamma$, induces an epimorphism of the KBSM, $j_{*}: \mathcal{S}(M-D) \rightarrow \mathcal{S}\left(M_{\gamma}\right)$.

Proof: The regular neighborhood, $V_{D}=[-1,1] \times D$, of $D$ in $M$ can be projected into 2-disk $D_{p}=[-1,1] \times[0,1]$ (then $\left.V_{D}=D_{p} \times[0,1]\right)$, and we use $D_{p}$ to present link diagrams, compare Fig.5.1. In $\mathcal{S}\left(M_{\gamma}\right)$ one has sliding relations described in Fig. 5.1 (with blackboard framing). These relations can be written as $p\left(z_{k}\right)=\left(-A^{2}-A^{-2}\right) z_{k}$, where $z_{k}$ is a link in $M$ in general position with $D$ and cutting it $k$ times; Fig.5.1. After simplifying the formula, using the Kauffman bracket skein relations, one gets: $p\left(z_{k}\right)=\left(-A^{2 k+2}-A^{-2 k-2}\right) z_{k}+\Sigma_{i=0}^{k-2} w_{i}(A) z_{i}$ and finally

$$
\left(A^{2 k+4}-1\right)\left(A^{2 k}-1\right) z_{k}=A^{-2 k-2} \sum_{i=0}^{k-2} w_{i}(A) z_{i}
$$

that is $\left(A^{2 k+4}-1\right)\left(A^{2 k}-1\right) z_{k}$ is a linear combination of links with a smaller than $k$ intersection number with the 2 -sphere $D_{\gamma}$. For $\left(A^{2 k+4}-1\right)\left(A^{2 k}-1\right)$ invertible in $R$, one can eliminate $z_{k}$ from the set of generators. Using induction, one can eliminate all elements of $\mathcal{S}\left(M_{\gamma}\right)$ which cut the 2 -sphere $D_{\gamma}$ non-trivially. Thus $j_{*}$ is an epimorphism. 


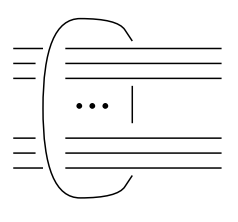

$$
\mathrm{p}\left(\mathrm{z}_{\mathrm{k}}\right)
$$

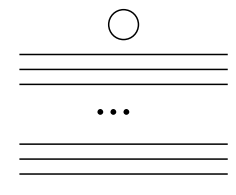

$\mathrm{z}_{\mathrm{k}} \cup \bigcirc$

Fig. 5.1.

Corollary 5.2 If $R$ is a ring with $\left(A^{k}-1\right)$ invertible for any $k>0$ then the embedding $j: M_{1} \# D^{3} \sqcup M_{2} \# D^{3} \rightarrow M_{1} \# M_{2}$ induces an epimorphism of the KBSM: $j_{*}: \mathcal{S}\left(M_{1} \# D^{3} \sqcup\right.$ $\left.M_{2} \# D^{3}\right) \rightarrow \mathcal{S}\left(M_{1} \# M_{2}\right)$.

\section{Proof of the main theorem}

We start the proof by showing that handle sliding described in Fig. 5.1 generates all handle sliding relations as long as $\left(A^{k}-1\right)$ is invertible for any $k>0$. This allows us to prove the main theorem for handlebodies as we know the basis of the KBSM in this case so we are able to choose only those handle slidings which reduce those links from the basis which cut $S^{2}$ (from the connected sum) non-trivially. Finally we prove the main theorem for any compact 3-manifold using the fact that such a manifold is obtained from a handlebody by adding 2 and 3-handles.

We say that a handle sliding $s l_{\gamma}$ of a link $L$ in $M$ along $\gamma \in \partial M$ has support in a submanifold $V$ of $M$ if $\gamma \in V$ and $L$ and $s l_{\gamma}(L)$ are identical outside $V$.

Lemma 6.1 Let $D$ be a meridian disk in $M, \gamma=\partial D$, and let $V_{D}=[-1,1] \times D$ be a regular neighborhood of $D$ in $M$.

(a) If a link $L$ is disjoint from the disks $\{-1,1\} \times D$ and the sliding sl $_{\gamma}$ has support in $(-1,1) \times D$, then the relation $L=\operatorname{sl}_{\gamma}(L)$ holds in $\mathcal{S}(M)$.

(b) Let $M_{0}$ be a component of $M$ which contains $D$ and assume that $L \cap M_{0}$ is a trivial knot, then the sliding relation $L=\operatorname{sl}_{\gamma}(L)$ holds in $\mathcal{S}(M)$ for any sliding of $L$.

(c) Let $L_{f r}^{\text {gen }}$ be a set of links generating $\mathcal{S}(M)$ and for each $L$ in $L_{f r}^{\text {gen }}$ choose a representative $D_{L}$ embedded in $M$ in such a way that $D_{L}$ cuts the meridian disk $D$ in the minimal number of points in its ambient isotopy class. Let $I_{0}$ be a submodule of $\mathcal{S}(M)$ generated by sliding relations of Fig. 5.1, for $z_{k}=D_{L}, L \in L_{f r}^{\text {gen }}$. In other words $I_{0}$ is generated by expressions $p\left(D_{L}\right)+\left(A^{2}+A^{-2}\right) D_{L}$. Then for any representative,

\footnotetext{
${ }^{2}$ Here we consider the concrete realization of links not ambient isotopy class. To omit confusion, we often will write $D_{L}$ for a representative of the ambient isotopy class of a link $L$.
} 
$\tilde{D}_{L}$, of a link $L$ in $M$ a sliding of Fig. 5.1 preserves the element of $\mathcal{S}(M) / I_{0}$ (i.e. $\left.\left(p(L)+\left(A^{2}+A^{-2}\right) L\right) \in I_{0}\right)$.

(d) If $R$ is a ring with $\left(A^{k}-1\right)$ invertible for any $k$ and $I_{0}$ is defined as in (c) then any sliding relation holds in $\mathcal{S}(M) / I_{0}$.

Proof:

(a) Because $V_{D}$ is a 3 -disk and $\left(V_{D}\right)_{\gamma}$, obtained from $V_{D}$ by adding a 2-handle along $\gamma$, is a 3-disk with a hole, therefore adding this 2-handle does not change the KBSM (Lemma2.1(2)(ii)). Therefore in $\mathcal{S}\left(V_{D}\right)$ and $\mathcal{S}(M-\{-1,1\} \times D)$ any relation of the form $L=s l_{\gamma}(L)$ holds. The embedding $i:(M-\{-1,1\} \times D) \rightarrow M$ induces the homomorphism of the KBSM $i_{*}: \mathcal{S}(M-\{-1,1\} \times D) \rightarrow \mathcal{S}(M)$, thus $i_{*}(L)=$ $i_{*}\left(s l_{\gamma}(L)\right)$. Lemma 6.1 follows, as assumptions of the lemma are chosen in such a way that any allowed sliding in $M$ is also a sliding in $M-(\{-1,1\} \times D)$.

(b) $L \cap M_{0}$, being a trivial knot, can be isotoped into $V_{D}$ without changing an ambient isotopy type of the result of the sliding, which will be also a trivial knot by part (a) of the lemma.

(c) Any link $L$ in $M$ can be written in $\mathcal{S}(M)$ as a linear combination of elements of $L_{f r}^{g e n}$. A sliding described in Fig. 5.1 does not depend on the presentation of $L$ (or elements of $L_{f r}^{g e n}$ ) so the lemma folllows. Notice that the sliding relation of Fig. 5.1 performed on the link $D_{L}$ disjoint from $D$ holds already in $\mathcal{S}(M)$.

(d) Let $D_{L}$ be a realization of a link $L$ in $M$. As $D_{L}$ is arbitrary, one can assume that sliding has support in $V_{D}$. Using relations from $I_{0}$ and the conclusion of the part (c) of the lemma together with Theorem 5.1 we can see that our slidings are performed on links in $M-\{-1,1\} \times D$ and have support in $V_{D}$, so by (a) of the lemma they do not introduce any new relation.

To visualize the assertion that modulo $I_{0}$ we need to slide only links $D_{L}$ in $M-$ $\{-1,1\} \times D$ with sliding support in $V_{D}$, consider disks $D_{1}=\{-1\} \times D$ and $D_{2}=$ $\{1\} \times D$ with $\gamma_{i}=\partial D_{i}$. Let $D_{L}$ be an arbitrary link in $M$ and $s l_{\gamma}$ a sliding with support in $\operatorname{int}\left(V_{D}\right)$. Slidings along $\gamma_{1}$ and $\gamma_{2}$ of the type described in Fig. 5.1, yield relations in $\mathcal{S}(M)$ satisfied in $\mathcal{S}(M) / I_{0}$ (as $\gamma_{i}$ is parallel to $\gamma$ ). These slidings allow us to reduce $D_{L}$ to a linear combination of links (curve systems) in $M-D_{1}-D_{2}$. Furhermore the support of sliding $s l_{\gamma}$ is (unchanged) in $\operatorname{int}\left(V_{D}\right)$.

As a corollary we get the main theorem for handlebodies. 


\section{Corollary 6.2 (Main theorem for handlebodies)}

Let $D$ be a meridian disk of a handlebody $H_{n}, \gamma=\partial D$. If $R$ is a ring with $\left(A^{k}-1\right)$ invertible for any $k$ then the embedding $j:\left(H_{n}-D\right) \rightarrow\left(H_{n}\right)_{\gamma}$ induces an isomorphism

$$
j_{*}: \mathcal{S}\left(H_{n}-D\right) \rightarrow \mathcal{S}\left(\left(H_{n}\right)_{\gamma}\right)
$$

Proof: By the handle sliding lemma (Lemma 4.1) one has $\mathcal{S}\left(\left(H_{n}\right)_{\gamma}\right)=\mathcal{S}\left(H_{n}\right) / J$ where $J$ is the submodule of $\mathcal{S}\left(H_{n}\right)$ generated by slidings $L-s l_{\gamma}(L)$. By Lemma $6.1 J=J_{0}$ for any generating set $L_{f r}^{g e n}$ of $\mathcal{S}\left(H_{n}\right)$. We can assume that $H_{n}=P_{n} \times[0,1]$ and $D=C \times[0,1]$ for an $\operatorname{arc} C$ properly embedded in the disk with $n$ holes, $P_{n}$. Let $B\left(P_{n}\right)$ be the basis of $\mathcal{S}\left(H_{n}\right)$ as described in Proposition 2.1(5). Let $B_{i}\left(P_{n}\right)$ be a subset of $B\left(P_{n}\right)$ composed of links with geometric intersection number with $C$ equal to $i$. By Lemma 6.1, $J_{0}$ is generated by sliding relations of Fig. 5.1, one relation for each element of $B_{i}\left(P_{n}\right)$ for $i>0$. Because $B\left(P_{n}\right)$ is a basis of $\mathcal{S}\left(H_{n}\right)$, therefore $B_{0}\left(P_{n}\right)$ is a basis of $\mathcal{S}\left(H_{n}\right) / J_{0}$. On the other hand $B_{0}\left(P_{n}\right)=B\left(P_{n}-C\right)$ is also a basis of $\mathcal{S}\left(H_{n}-D\right)$, thus $j_{*}$ is an isomorphism.

We are ready now to prove the main theorem.

Theorem 6.3 Let $D$ be a meridian disk of $M$ and $\gamma=\partial D$. If $R$ is a ring with $\left(A^{k}-1\right)$ invertible for any $k$ then the embedding $j:(M-D) \rightarrow M_{\gamma}$ induces an isomorphism

$$
j_{*}: \mathcal{S}(M-D) \rightarrow \mathcal{S}\left(M_{\gamma}\right)
$$

Proof: $M_{\gamma}$ can be obtained from $\left(H_{n}\right)_{\gamma}$ by adding to $M_{\gamma}$ some 2-handles (disjoint from the 2-handle added along $\gamma$ ) and some 3-handles. By Lemma 4.1 and Proposition 2.1(2) $\mathcal{S}\left(M_{\gamma}\right)$ is obtained from $\mathcal{S}\left(\left(H_{n}\right)_{\gamma}\right)$ by sliding links generating $\mathcal{S}\left(\left(H_{n}\right)_{\gamma}\right)$ along these 2-handles. Denote these slidings by $s l_{h}$. Consider any link $L$ in $\left(H_{n}\right)_{\gamma}$ and any sliding $s l_{h}$. We can choose a representative $D_{L}$ of $L$ so that $D_{L}$ and $s l_{h}\left(D_{L}\right)$ are identical in the neighborhood of $S^{2}=D_{\gamma}$. By Lemma 6.1 we can present $D_{L}$ in $\mathcal{S}\left(\left(H_{n}\right)_{\gamma}\right)$ as a linear combination of links which are disjoint from $S^{2}$ and differ from $D_{L}$ only in a small neighborhood of $S^{2}$. Thus the sliding relation $D_{L}-s l_{h}\left(D_{L}\right)$ is a linear combination of sliding relations in $\left(H_{n}\right)_{\gamma}-S^{2}$. Therefore $j_{*}: \mathcal{S}(M-D) \rightarrow \mathcal{S}\left(M_{\gamma}\right)$ is an isomorphism. The proof of Theorem 6.3 is complete.

Corollary 6.4 Let $R$ be a ring with $\left(A^{k}-1\right)$ invertible for any $k$ then

(i) If $S^{2}$ is a 2-sphere embedded in $M$ then the embedding $j: M-S^{2} \rightarrow M$ yields an isomorphism of the $K B S M j_{*}: \mathcal{S}\left(M-S^{2}\right) \rightarrow \mathcal{S}(M)$.

(ii) The embedding $M_{1} \# D^{3} \sqcup M_{2} \# D^{3}$ to $M_{1} \# M_{2}$ yields an isomorphism of the KBSM. 
(iii) $\mathcal{S}\left(M_{1} \# M_{2}\right)$ is isomorphic to $\mathcal{S}\left(M_{1}\right) \otimes \mathcal{S}\left(M_{2}\right)$.

(iv) $\mathcal{S}\left(S^{1} \times S^{2}\right)=R$

Proof: The case (i) follows immediately from Theorem 6.3. The case (ii) corresponds to the case of (i) when $S^{2}$ separates $M . M_{1} \# M_{2}-S^{2}$ and $M_{1} \# D^{3} \sqcup M_{2} \# D^{3}$ differ only by parts of their boundaries so their KBSM are the same. The case (iii) follows from (ii) by Proposition 2.1(3). The case (iv) follows from (i) as $S^{1} \times S^{2}-S^{2}$ is a 3-disk with two holes. The case (iv) is also a special case of a general theorem in [H-P-2].

Corollary 6.5 If $S^{2}$ is a 2-sphere embedded in $M$ and the $K B S M \mathcal{S}\left(M-S^{2} ; Z\left[A^{ \pm 1}\right], A\right)$ is free then the embedding $j: M-S^{2} \rightarrow M$ yields a monomorphism of the $K B S M j_{*}$ : $\mathcal{S}\left(M-S^{2} ; Z\left[A^{ \pm 1}\right], A\right) \rightarrow \mathcal{S}\left(M ; Z\left[A^{ \pm 1}\right], A\right)$.

Proof: Let the set $\left\{x_{\alpha}\right\}$ be a basis of $\mathcal{S}\left(M-S^{2} ; Z\left[A^{ \pm 1}\right], A\right)$. By the Universal Coefficient Property it is also a basis of $\mathcal{S}\left(M-S^{2} ; \mathcal{F}(A), A\right)$. By Theorem 6.3 the set $\left\{j_{*}\left(x_{\alpha}\right)\right\}$ is a basis of $\mathcal{S}(M ; \mathcal{F}(A), A)$. Therefore it is a $Z\left[A^{ \pm 1}\right]$ linearly independent set in $\mathcal{S}\left(M ; Z\left[A^{ \pm 1}\right], A\right)$, and therefore $j_{*}$ is a monomorphism.

\section{Generalizations and Speculations.}

Theorem 1.2 does not hold for the ring $R=Z\left[A^{ \pm 1}\right]$. As observed in [P-2], Theorem 4.4, $\mathcal{S}\left(M_{1} \# M_{2} ; Z\left[A^{ \pm 1}\right], A\right)$ often contains a torsion part. The general description (generators and relators) of the KBSM is possible by Theorem 4.1 but to have a more meaningful description one should first analyze relative KBSM in manifolds $M_{1}$ and $M_{2}$ and the KBSM of the connected sum would be the sum of tensor products of "reduced" relative skein modules. Finally one should be able to obtain for KBSM a Van Kampen-Seifert type theorem for 3-manifolds (glued along surfaces) \$. The theorem could be reminiscent of Topological Quantum Field Theory formalism At]. We plan to give the detailed description of the KBSM of connected and disc sums of 3-manifolds in $\mathrm{P}-4]$. Here we quote one, relatively simple result (where there is no need to invoke the notion of the relative KBSM).

\section{Theorem 7.1}

$$
\mathcal{S}\left(H_{n} \# H_{m}\right)=\mathcal{S}\left(H_{n+m}\right) / \mathcal{I}
$$

where $\mathcal{I}$ is the ideal generated by expressions $z_{k}-A^{6} u\left(z_{k}\right)$, for any even $k \geq 2$, and $z_{k} \in$ $B_{k}\left(P_{n+m}\right)$, where $B_{k}\left(P_{n+m}\right)$ is a subset of a basis $B\left(P_{n+m}\right)$ composed of links with geometric intersection number with a disk $D$ separating $H_{n}$ and $H_{m}$ equal to $k . u\left(z_{k}\right)$ is a modification

\footnotetext{
${ }^{3}$ The recent paper by W.Lofaro is a step in this direction Lof.
} 
of $z_{k}$ in the neighborhood of $D$, as shown in Fig. 7.1. Our relation $z_{k}=A^{6} u\left(z_{k}\right)$, is a result of the sliding relation $z_{k}=s l_{\partial D}\left(z_{k}\right)$ as illustrated in Fig. 7.2.
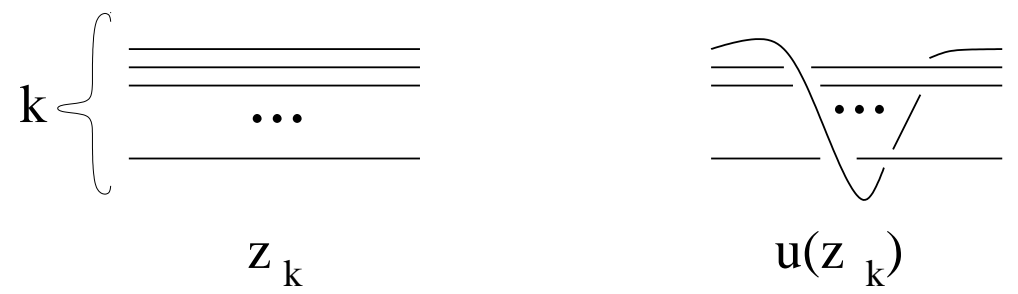

Fig. 7.1.
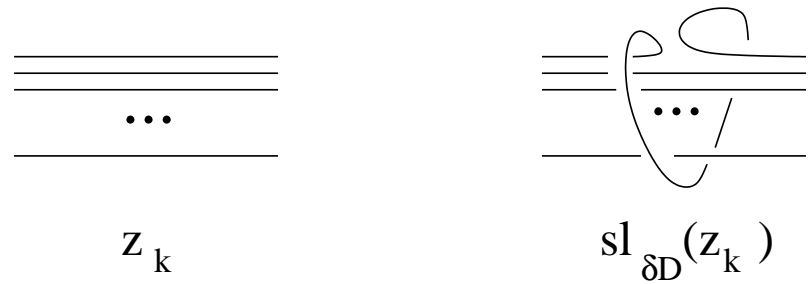

Fig. 7.2.

\section{References}

[At] M.Atiyah, The geometry and physics of knots. Lezioni Lincee (Lincei Lectures), Cambridge University Press, Cambridge, 1990. x+78 pp.

[Bl] T.S. Blyth, Module theory, Clarendon Press, Oxford 1977.

[C-E] H.Cartan, S.Eilenberg, Homological Algebra, Princeton University Press, 1956.

[H-P-1] J. Hoste, J.H. Przytycki, A survey of skein modules of 3-manifolds; in Knots 90, Proceedings of the International Conference on Knot Theory and Related Topics, Osaka (Japan), August 15-19, 1990, Editor A. Kawauchi, Walter de Gruyter 1992, 363-379.

[H-P-2] J. Hoste, J.H. Przytycki, The Kauffman bracket skein module of $S^{1} \times S^{2}$, Math. Z., 220(1), 1995, 63-73.

[Lof] W.F.Lofaro, A Mayer-Vietoris theorem for the Kauffman bracket skein module, Journal of Knot Theory and Its Ramifications, 8(6), 1999, 721-729.

[P-1] J.H. Przytycki, Skein modules of 3-manifolds, Bull. Polish Acad. Science, 39(1-2), 1991, 91-100. 
[P-2] J.H. Przytycki, Algebraic topology based on knots: an introduction, Knots 96, Proceedings of the Fifth International Research Institute of MSJ, edited by Shin'ichi Suzuki, 1997 World Scientific Publishing Co., 279-297.

[P-3] J.H. Przytycki, Fundamentals of Kauffman bracket skein modules, Kobe Math. J., 16(1), 1999.

[P-4] J.H. Przytycki, Algebraic topology based on knots, Series on Knots and Everything, Vol. 18, World Scientific Publishing Co., in preparation.

[P-S] J.H. Przytycki, A.S. Sikora, Skein algebra of a group, Banach Center Publications, Vol. 42, Knot Theory, 1988, 297-306.

$$
\begin{gathered}
\text { Department of Mathematics, University of Maryland } \\
\text { College Park, MD 20742 }
\end{gathered}
$$

The author is on leave from:

$$
\begin{gathered}
\text { Department of Mathematics, The George Washington University } \\
\text { 2201 G Str. Funger Hall } \\
\text { Washington, D.C.20052 } \\
\text { przytyck@gwu.edu }
\end{gathered}
$$

